electric stimulation (isolated $10 \mathrm{~V} ., 1 \mathrm{msec}, 50 \mathrm{c} / \mathrm{s}$ ) in the concentric bipolar needle electrode placed stereotaxically to the ventro-lateral portion of the left thalamus. When the stimulation stopped, the abnormal movement stopped. The $6 \mathrm{c} / \mathrm{s}$ electric stimulation of this condition induced $6 \mathrm{c} / \mathrm{s}$ brain waves in the bilateral frontal and central leads.

This point was the mid-point of a line from the posterior margine of the foramen of Monro to the center of pineal calcification on the lateral projection radiograph. On the A-P projection radiograph, it was at the level of the floor of the lateral ventricle and within a vertical line dropped from the lateral most part of the body of the lateral ventricle.

Immediately after the injection of $0.3 \mathrm{ml}$ of alcohol-caoline-Myodil paste in this point, the choreoid movement was abolished without any disability from this procedure. Since then the patient has had no choreoid or ballistic movement of the right limbs for 6 months. (Presented $8 \mathrm{~mm}$ movie of pre- and postoperative status of the patient.)

\title{
18. Effect of Combined Thalamic Nucleic Destruction on Spasmodic Torticollis
}

\author{
Nobuo YoshiI, Shiro Shimizu, Yoshiyuki IsHIDA, \\ Mizuo Kagawa and Kimihiro MinaKami \\ Neurological Surgery, Keio University Hospital
}

\section{Chemothalamectomy for 3 Cases with Myoclonus}

\author{
Yutaka Hori, Shozaburo Utsumi, Chikayoshi Terada, Seiji Miyamoto, \\ Yoshiaki, Ueda, Katsuya Shrota and Shigeki Minamr \\ 2nd Dept. of Surgery, Nara Medical Univ.
}

For the past 5 years we have performed stereotaxic operation for 3 cases with myoclonus. It was assumed that these cases did not belong to category of myoclonic epilepsy, but to diseases of extrapyramidal system.

Case 1. A male, aged 19 years, complained of muscle twitching evoked by voluntary effort in the left upper extremity since one year ago. On January, 1963, thalamectomy (Right-V.o.p., CM) was performed and then muscle twitching diminished markedly in severity, but not completely, sometimes evoked by voluntary efforts. One year later, muscle twitching disappeared completely, and then 
3 years after operation the patient was doing well and went abroad.

Case 2. A male, aged 28 years, had complained of muscle twitching in the left upper extremity and shoulder, soon after appeared in the right side.

On admission, muscle twitching was demonstrated in the right side more markedly than in the opposite side. On January, 1967, thalamectomy (LeftV.o.p.) was performed, and the patient had marked improvement in clinical feature.

Six month after surgery, contralateral thalamectomy (Right-V.o.p.) was done, and effective results was obtained.

Case 3. A male, aged 21 years, had a history of the sudden onset of muscle twitching after having high fever for one night, and this twitching had spread to all body after one year. It was thought that muscle twitching was ressemble to clonic convulsion at the most severe stage, but no spike or spike \& wave complex was found in frequent electroencephalographical recording. On August 19, 1966, thalamectomy (Left-V.o.p.) was performed and muscle twitching was suppressed fiarly, he could so much as speak a little. Six month after the first operation, contralateral thalamectomy (Right-V.o.p.) was done, muscle twitching was exag. gerated temporally, but after one year later, the patient had marked improvement from his myoclonus.

His myoclonic attack was evoked by visual stimulation, so patient closed his eyes with towel before surgery. This patient had been in hospitality untl present time, doing well without towel, and could speak out simple words. 